\title{
Letter Editorial: Rehabilitation Services: Why Should We Use Tele-Rehabilitation in Iran? Necessity and Application
}

\author{
Niloufar Rabanifar ${ }^{1}$ (D), Kianoush Abdi $^{1^{*}}$ (D) \\ 1. Department of Rehabilitation Management, University of Social Welfare and Rehabilitation Sciences, Tehran, Iran.
}

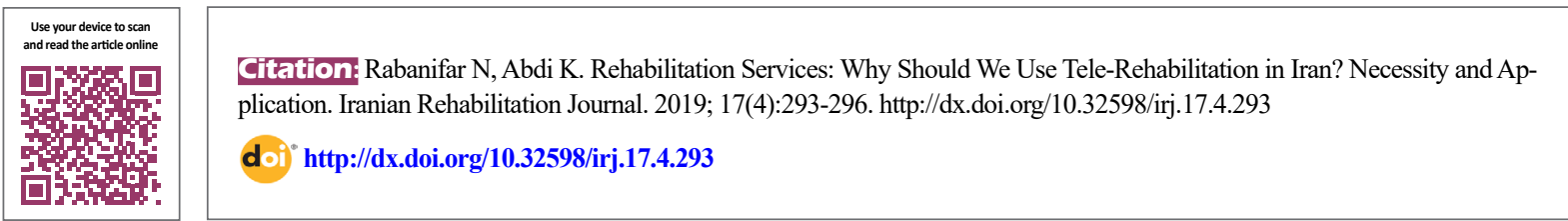

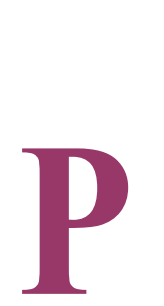

\section{Dear editor-in-chief}

eople living in rural areas far from health care centers are more likely to require health care services because of their long-distance services and poverty [1]. Tele-rehabilitation is considered as one of the rapidly emerging and growing areas of telemedicine and a precious model for providing clinical services like assessment, treatment, and follow-ups, as well as reducing the length of time for hospitalization. In fact, this method can have similar or even better results compared to conventional interventions [2] and sometimes can be used as an alternative for ordinary services [3].

\section{Why Tele-rehabilitation?}

In the conventional rehabilitation approaches based on care centers or hospitals, the patient should pass a distance for receiving treatment that requires wasting time and cost $[2,4]$. Distance, insufficient resources, and mobility challenges may hinder access to evidence-based treatment [5], while Tele-rehabilitation can overcome these barriers and decrease the fatigue and non-discipline of traditional treatment sessions [2]. On the other hand, people involved in this area are helped by reducing the sense of isolation among the rural therapists, providing indirect training to them, and improving the stability in the areas with a high turnaround of therapists [6]. Tele-rehabilitation has considerable advantages, especially in telecommunications development. Communications development transfers the medical information and is of great importance for the patients, their families, caregivers, and therapists [7]. Telepractice can be a link between the family and the therapy team. Providing Tele-rehabilitation not only decreases the travel and its related problems but also makes the families focus more comfortably on the needs of their children. In addition, it decreases the dependency of the family on the therapist and enables the family to get involved with the treatment process directly [8]. This approach can have a positive effect on capacity, quality of life, self-efficacy, and commitment to people with disabilities [9]. Tele-rehabilitation can help the elderly to do their daily activities in order to improve their lifestyle and integrate them into society [3]. The development of technology, the Internet, the provision of minimum infrastructure, and the equipment of providers and recipients with smartphones, even in the most remote areas, increase the necessity of attention.

\section{The Applications of Tele-rehabilitation}

Tele-rehabilitation can have different clinical applications, including the evaluation of needs and environmental-individual factors, diagnosis, treatment and intervention, consultation, follow-up, and even the training of

* Corresponding Author:

Kianoush Abdi, PhD.

Address: Department of Rehabilitation Management, University of Social Welfare and Rehabilitation Sciences, Tehran, Iran.

Tel: +98 (912) 7603800

E-mail:k55abdi@yahoo.com 
the involved therapists [6]. In addition, the specialized areas where this approach can provide services are very different, including physiotherapy, occupational therapy, speech therapy, vocational rehabilitation [6], cardiovascular, and neurological rehabilitation [10].

Other applications of Tele-rehabilitation include:

Consulting with specialists and rehabilitation engineers for guidance

Observing and evaluating the patient's condition

Evaluating the weaknesses and strengths of the patient's home structure and presenting suggestions and aids for better matching to the patient's needs

Presenting assistance technologies

Evaluating the ability to do work, social life, recreation, and individual independence in society

Controlling and managing the patients' bedsore by receiving high- or low-quality images from the patient

Telepractice by using tools like an electromyographycontrolled game for the rehabilitation of stroke patients or rewriting an interactive story for the patients suffering from brain damage.

Tele-rehabilitation management

And training the use of complementary communications equipment [6]

Tele-rehabilitation can influence the improvement of community-based rehabilitation, especially in developing countries, where community rehabilitation services are expensive and out of access [3]; particularly in Iran, where there are many barriers such as difficult access and financial problems and costs for providing rehabilitation services to people with disabilities [11]. However, before implementing this approach, it should be accepted by providers, recipients, and society [6]. Thus, since Telerehabilitation has many advantages and applications in providing rehabilitation services to people with disabilities, because of the negligence of this issue in Iran, it requires special attention to improve the provision of services for more access and increasing satisfaction.

\section{Ethical Considerations}

\section{Compliance with ethical guidelines}

There was no ethical considerations to be considered in this research.

\section{Funding}

This research did not receive any specific grant from funding agencies in the public, commercial, or not-forprofit sectors.

\section{Authors' contributions}

Idea, Conceptualization, Writing, Editing and Finalizing: Kianoush Abdi, Niloufar Rabanifar. Supervision and management: Kianoush Abdi.

\section{Conflict of interest}

The authors believe that there is no conflict of interest in this paper.

\section{References}

[1] Cason J. A pilot Tele-rehabilitation program: Delivering early intervention services to rural families. International Journal of Tele-rehabilitation. 2009; 1(1):29. [DOI:10.5195/ IJT.2009.6007]

[2] Rogante M, Kairy D, Giacomozzi C, Grigioni M. A quality assessment of systematic reviews on Tele-rehabilitation: What does the evidence tell us? SciELO Public Health. 2015; 51(1):11-8. [DOI:10.4415/ANN_15_01_04] [PMID]

[3] Zahid Z, Atique S, Saghir MH, Ali I, Shahid A, Malik RA. A commentary on Tele-rehabilitation services in pakistan: Current trends and future possibilities. International Journal of Tele-rehabilitation. 2017; 9(1):71-6. [DOI:10.5195/ IJT.2017.6224] [PMID] [PMCID]

[4] Covert LT, Slevin JT, Hatterman J. The Effect of Tele-rehabilitation on Missed Appointment Rates. International Journal of Tele-rehabilitation. 2018; 10(2):65-72. [DOI:10.5195/ IJT.2018.6258] [PMID] [PMCID]

[5] Benham S, Gibbs V. Exploration of the Effects of Telerehabilitation in a School-Based Setting for At-Risk Youth. International Journal of Tele-rehabilitation. 2017; 9(1):39-46. [DOI:10.5195/IJT.2017.6215] [PMID] [PMCID]

[6] Schmeler MR, Schein RM, McCue M, Betz K. Tele-rehabilitation clinical and vocational applications for assistive technology: Research, opportunities, and challenges. International Journal of Tele-rehabilitation. 2009; 1(1):59-72. [DOI:10.5195/ IJT.2009.6014] [PMID] [PMCID] 
[7] Barlow IG, Liu L, Sekulic A. Wheelchair seating assessment and intervention: A comparison between Tele-rehabilitation and face-to-face service. International Journal of Tele-rehabilitation. 2009; 1(1):17-28. [DOI:10.5195/IJT.2009.868] [PMID] [PMCID]

[8] Parfitt M, Ramachandar S, Ruth G. Shortening the Distance: Empowering Parents through Telepractice. Volta Voices. 2016; 23(2):1

[9] Tsai LLY, McNamara RJ, Dennis SM, Moddel C, Alison JA, McKenzie DK, et al. Satisfaction and experience with a supervised home-based real-time videoconferencing Tele-rehabilitation exercise program in people with Chronic Obstructive Pulmonary Disease (COPD). International Journal of Telerehabilitation. 2016; 8(2):27-38. [DOI:10.5195/IJT.2016.6213] [PMID] [PMCID]

[10] Peretti A, Amenta F, Tayebati SK, Nittari G, Mahdi SS. Telerehabilitation: Review of the State-of-the-Art and Areas of Application. JMIR Rehabilitation and Assistive Technologies. 2017; 4(2):e7. [DOI:10.2196/rehab.7511] [PMID] [PMCID]

[11] Abdi K, Arab M, Rashidian A, Kamali M, Khankeh HR, Farahani FK. Exploring Barriers of the Health System to Rehabilitation Services for People with Disabilities in Iran: A Qualitative Study. Electron Physician. 2015; 7(7):1476-85. [DOI:10.19082/1476] [PMID] [PMCID] 
This Page Intentionally Left Blank 